\title{
Assessment of spinal curvatures in static postures using localized 3D ultrasound: A proof-of-concept study
}

This paper was downloaded from TechRxiv (https://www.techrxiv.org).

LICENSE

CC BY-NC-SA 4.0

SUBMISSION DATE / POSTED DATE

$13-01-2022$ / 03-02-2022

\section{CITATION}

Laura;, Meszaros-Beller,; Antico, Maria; Fontanarosa, Davide; pivonka, peter (2022): Assessment of spinal curvatures in static postures using localized 3D ultrasound: A proof-of-concept study. TechRxiv. Preprint. https://doi.org/10.36227/techrxiv.18316922.v1

$\mathrm{DOI}$

10.36227/techrxiv.18316922.v1 


\title{
Assessment of spinal curvatures in static postures using localized 3D ultrasound: A proof-of-concept study
}

\author{
L. Meszaros-Beller ${ }^{1,2}$, M. Antico ${ }^{1,2}$, D. Fontanarosa ${ }^{2,3}$ and P. Pivonka ${ }^{1,2^{*}}$
}

\begin{abstract}
Measurement of vertebral spinal motion is a difficult endeavor given the lack of reachable bony landmarks for placement of reflective markers for common optoelectronic systems and large skin movement artifacts along the spine affecting the accuracy of surface-mounted techniques. The objective of this proof-of-concept study was to develop an experimental framework to assess spinal curvatures with high accuracy by using ultrasound (US) imaging. A phantom spine model (T1-T12) immersed in water was scanned using both Magnetic resonance imaging (MRI) and three-dimensional (3D) US. The US scans were obtained for the phantom spine in different postures while the US probe was localized by three electromagnetic tracking sensors (NDI system) attached to the probe head and later identified on the acquired US volumes via visual inspection. Spinal landmarks were selected on the US images and validated using US-MRI registration. Their position was then related to the position of the tracking sensors, enabling the precise localization of landmarks and reconstruction of spinal curvatures in a global reference frame. The obtained anatomical reconstructions were further refined using an automatic registration algorithm and validated by an experienced sonographer (ground-truth solution). Results of this study show that localized 3D US enables tracking of spinal landmarks and allows for an accurate reconstruction of static postures. A high reliability in sensor identification of $0.49 \mathrm{~mm}$ was found on average while the inter-observer variability was strongly correlated to the intra-observer error with maximum deviance of $0.8 \mathrm{~mm}$. The NDI-based spine reconstruction had a mean error of $5.6 \mathrm{~mm}$ compared to the ground-truth, which is significantly lower than errors obtained by current gold standards $(>10 \mathrm{~mm})$. Combining US probe tracking and semi-automatic registration methods anatomical reconstructions comparable to clinical standards were obtained. This study implies that US holds the potential for advancing vertebral motion assessment and the reconstruction of spinal curvatures. In future, this could overcome limitations of marker-based approaches.
\end{abstract}

Index Terms - 3D ultrasound, spinal landmarks, spinal reconstruction, spine phantom, vertebral motion

\section{INTRODUCTION}

A NALYSIS of spine biomechanical function requires accurate measurement of intervertebral motion. Currently, only radiological techniques based on X-ray imaging are able to measure intervertebral motion with high accuracy $[1,2,3]$. However, exposure to ionizing radiation poses health risks and is not ethical for use in a healthy population.

The authors wish to thank Marian Steffens for validating the registrations performed in this study and Christopher Edwards for medical advice giving with sonographic measurements. Laura Meszaros-Beller acknowledges the support by Queensland University of Technology (QUT), in the framework of the Clayton Adam Florence Wilson Award PhD scholarship for spinal research. Maria Antico was supported by the Centre for Biomedical Technologies.

*Corresponding author: P. Pivonka (email:peter.pivonka@qut.edu.au)
Marker-based optoelectronic approaches are considered the gold standard for accurately characterizing joint motion in the hip, knee and shoulder. For the spine, however, the latter fail to be sufficiently accurate due to large skin movement relative to the vertebral body [4,5]. Also, due to the fact that vertebral bodies are relatively short, small errors in marker location can lead to significant errors in defining vertebral body position [5]. The spine is a multi-joint system composed of individual functional spinal units (FSUs), so this error is likely to propagate and amplify over larger spinal segments such as the lumbar, the thoracic and the cervical region. Here we propose to use localized ultrasound (T-US) imaging to measure sagittal movement of vertebral bodies of the thoracic spine.

Measurement of intervertebral motion has numerous applications including diagnosing back pain, spinal deformity, disc degeneration, and biomechanical inverse kinematic calculations for musculoskeletal models of the spine. In a biomechanical context, angles between vertebrae in FSUs during motion tasks (e.g., flexion-extension) assessed using marker-based approaches are currently estimated as constant (population-based) fractions of the measured entire lumbar (or thoracic) segment angles [6,7]. However, concerns persist over the accuracy of this approach, neither does it allow for subjectspecific consideration of spinal biomechanics. Improvement in accuracy of intervertebral motion tracking will ultimately result in more accurate inverse kinematic data which can then be subsequently used for inverse dynamics calculations of FSUjoint forces, FSU-moments and spinal muscle forces [8]. This will also help improve current design of spinal implants such as artificial disc implants, and hopefully make vertebral fusion a procedure of the past, given the high risks associated with this technique [9]. Furthermore, having access to intervertebral motion could help explore the relation among muscle dysfunction, reduced proprioception and low back pain, and could therefore inform rehabilitation [10].

The use of sonographic systems for tracking of spinal posture and motion has been largely driven by the clinical need to quantify spinal deformity and surgical navigation, and more recently by biomechanical needs to more accurately measure spinal kinematics. Chen et al. and Suzuki et al. reported on accurate measurement of spinous processes and laminae as

\footnotetext{
1 School of Mechanical, Medical and Process Engineering, Queensland University of Technology, Brisbane, Queensland 4000, Australia

2 School of Mechanical, Medical and Process Engineering, Queensland University of Technology, Brisbane, Queensland 4000, Australia

${ }^{3}$ School of Clinical Sciences, Queensland University of Technology, Brisbane, Queensland 4000, Australia
} 
$>$ REPLACE THIS LINE WITH YOUR MANUSCRIPT ID NUMBER (DOUBLE-CLICK HERE TO EDIT) <

landmarks for characterizing spinal deformities using ultrasound (US) imaging [11,12]. Tracking of vertebral bodies using two-dimensional (2D) T-US in static positions has previously been used for reconstructing the spine anatomy and measuring in-plane spinal curvature in scoliosis patients $[13,14,15,16]$. Those studies have shown that US measures of 2D curvature are comparable with those obtained using X-ray based imaging methods and, consequently, could replace the latter methods. While these results are promising, no attempts have been made for accurate reconstruction and landmark identification and tracking from 3D T-US imaging.

The study of van den Horn validated an US imaging technique to measure sagittal plane lumbosacral motion [17]. Lumbosacral angle change was measured and validated using direct and indirect measures. The method was tested on an invitro human spine and on an in-vivo porcine spine and validated against video and fluoroscopy measurements. Recently, Bringol and co-workers proposed an automated method for vertebral landmark detection from US images [18]. Results indicated that spinous processes can be detected with high accuracy, while detection of laminae is challenging. More recently, a dynamic US tracking system using A-mode was developed by Niu and co-workers for the assessment of tibiofemoral kinematic [19]. It was demonstrated that the T-US system showed lower kinematic errors compared to markerbased systems, with bone pins providing ground-truth data $[19,20]$. Masum et al. utilized tri-plane B-mode ultrasound for 3D kinematic analysis of knee joints [21].

In this work, we explored the possibility to use 3D T-US, localized using commercially available electromagnetic sensors, to detect relevant landmarks on the spine. Multiple automatically registered volumetric scans were used to describe a thoracic phantom spine, in three configurations, i.e., in different curvature poses. The method was cross-validated using medical image processing techniques and a Magnetic resonance imaging (MRI) scan of the same phantom. It is already currently possible to envision real-time tracking of the whole spine while the curvature changes, as long as the motion is sufficiently slow compared to the US probe refresh rate, potentially enabling advanced insight into internal kinematics, which could overcome the limitations of current gold standards.

\section{MATERIALS AND METHODS}

In the present proof-of-concept study, we explored the potential of US imaging for the assessment of vertebral spinal motion on a benchmark level. We hypothesize that (i) 3D T-US allows tracking of spinal landmarks in a global reference frame and (ii) that 3D T-US is suitable for accurate reconstruction of spinal anatomy. To evaluate the feasibility of the proposed methodology, a phantom model of the thoracic spine consisting of individual vertebrae (T1-T12) connected by a string and intermediate rubbery intervertebral discs was used.

\section{A. Data collection}

MRI Segmentation

A Siemens Magnetom Prisma 3T MRI System was used to image the full spine phantom immersed in water in a straight posture, i.e., with the vertebral levels aligned. In the obtained MRI scan, the bony-surrogate of the spine was represented by dark pixel intensities and was segmented from the surrounding water, represented by light pixel intensities, using an automatic threshold algorithm in 3DSlicer v4.10.2.

\section{US imaging and localization of landmarks}

US scans were performed on the spine phantom immersed in a water tank using a 3D US transducer (Philips VL 13-5) and the Philips Epiq7 system. The settings of the Philips system optimized for the visualization of the phantom model included: 4-6 cm penetration depth; emission power of $-0.5 \mathrm{~dB}$; far field focus; dynamic range of $60 \mathrm{~dB}$; medium persistence; wide scan; SonoCT real-time compound imaging technology and XRES image processing. The volume field of view of the probe was $38 \mathrm{~mm} \times 30^{\circ}$.

The spine phantom, reinforced by a flexible rod, was clamped onto a stable L-shaped base within the water tank and brought into three static configurations (i.e., poses) $\operatorname{pos}_{i}$ with $i=1,2,3$ in the sagittal plane by stepwise bending the model from an initially straight $\left(\operatorname{pos}_{1}\right)$ to a curved configuration $\left(\operatorname{pos}_{2}, \mathrm{pos}_{3}\right)$ mimicking a spinal flexion task. For the sake of simplicity, we will further refer to the 'straight', 'curved' and 'curved 2' configuration. Each configuration $\operatorname{pos}_{i}$ was captured by collecting 3D T-US volumes of every vertebral level between T1-T5, translating the probe along the spinal processes of the phantom by a few centimeters while keeping the transducer head parallel to the spine. During data collection, the US probe was connected to a probe holder providing stability, and was localized using a commercial electromagnetic tracking system (NDI Aurora). Three NDI tracking sensors were attached to the convex beam of the probe head using adhesive tape in order to localize the probe in space (Fig. 1). Within the US volume created by the 3D US transducer, every voxel was then related to the location of the tracking sensors, as reported in detail in Section II-C. An illustration of the experimental data collection is shown in Fig. 2.

\section{B. US-based landmark identification \& MRI-based validation}

Commonly, a minimum of three anatomical landmarks are selected for the precise localization and scaling of a body segment. Previous studies have shown that US imaging allows for the visualization of the posterior vertebral surface together with the identification of specific landmarks [11,18]. Particularly, the tip of the spinous process (Fig. 3A-1) and the laminae of the vertebral arch (Fig. 3A-2) create areas of hyperechoic reflection in US. From a biomechanical point of view, the posterior vertebral surface, hence, provides sufficient information in order to localize the vertebra in space.

A triplet of US-derived landmarks $L^{U S}$, i.e., spinous process (SP), left lamina (LL) and right lamina (LR), were selected in voxel coordinates for every vertebra T1-T5 in the 'straight' configuration using ImFusion, a commercial software for the visualization of medical images. Prior to that, each US volume 
$>$ REPLACE THIS LINE WITH YOUR MANUSCRIPT ID NUMBER (DOUBLE-CLICK HERE TO EDIT) <

was registered manually to the MRI of the phantom spine in order to cross-validate the US-based selection of landmarks. Note, each US-MRI registration performed in this study was done manually and verified by an experienced sonographer. In Fig. 3B the US-MRI registration for vertebra T2 is visualized for all configurations $\operatorname{pos}_{i}$. Landmarks for the curved configurations $\left(L_{\text {pos }_{2}}^{U S}, L_{\text {pos }_{3}}^{U S}\right)$ were derived from those selected in the straight configuration ( $\mathrm{L}_{\text {pos }_{1}}^{\mathrm{US}}$ ) by mapping the landmarks $\mathrm{SP}, \mathrm{LL}, \mathrm{LR} \in \mathrm{L}_{\mathrm{pos}_{1}}^{\mathrm{US}}$ to the US volumes of the curved configurations, as in (1). This ensured that identical landmarks were tracked in all configurations $\operatorname{pos}_{i}$.

$$
L_{\text {pos }_{1}}^{U S} \rightarrow L_{\text {pos }_{2}}^{U S}, L_{\text {pos }_{3}}^{U S}
$$

Sensor positions $S_{\text {pos }_{i}}^{U S}$ in voxel coordinates were selected individually for each US volume and configuration $\operatorname{pos}_{i}$ through visual inspection in ImFusion. Since all three sensors were positioned perpendicular to the US probe's axial plane, the sensor appeared as a hyperechoic circular shape along the former plane. Starting from the first axial image where the sensor connection with the wire was visible and scrolling through the volume, the sensor tip coordinates were identified in the last axial image where the sensor was visible. The sensor identification was confirmed by visualizing the sensor in the sagittal and coronal plane of the volume and the $3 \mathrm{D}$ rendering provided by the software.

\section{Localization of the US volumes in global coordinates}

During the US data collection, three sensors were attached to the probe head and tracked through the NDI tracking system, as elucidated in Section II-A. Thus, for each acquired volume, the sensor tip coordinates of the three sensors were obtained relative to the NDI system, as in (2).

$$
S_{i}^{N D I}=\left(x_{i}, y_{i}, z_{i}\right)
$$

where the subscript $i=1,2,3$ corresponds to the sensor ID and $x, y, z$ to the sensor coordinates in the NDI coordinate system. The sensors' tip coordinates were defined in the US coordinate system by selecting the center of the middle pixel of the US volume as the origin of this coordinate system and computing the location of each sensor, whose voxel coordinates were identified as described in Section II-B, in $\mathrm{mm}$ using voxel spacing of the US volume, as in (3).

$$
S_{i}^{U S, w}=\left(x^{\prime}{ }_{i}, y^{\prime}{ }_{i}, z^{\prime}{ }_{i}\right)
$$

where the subscript $i=1,2,3$ corresponds to the sensor ID and $x^{\prime}, y^{\prime}, z^{\prime}$ to the sensor coordinates in the US coordinate system. The homogeneous transformation from US to NDI representation ${ }_{N D I} T^{U S}$ was obtained for each US volume using the 'absor' function, a least square regression method, implemented in MATLAB [22], finding the 3D rotation matrix $\left(R_{3 \times 3}\right)$ and translation vector $\left(t_{3 \times 1}\right)(4)$ that minimises the sum of squared distances between the two sets of datapoints corresponding to the sensor locations in the NDI $\left(S_{i}^{N D I}\right)$ and in the US coordinate system $\left(S_{i}^{U S, w}\right)$, respectively (5).

$$
S_{i}^{N D I}={ }_{N D I} T^{U S} S_{i}^{U S, w}
$$

with ${ }_{N D I} T^{\text {US }}=\left[\right.$ Rt $\left.0_{1 \times 3} 1\right]$

$$
\sum^{i}\left\|R S_{i}^{U S, w}+t-S_{i}^{N D I}\right\|^{2}
$$

where $S_{i}^{U S, w}=\left[S_{1}^{U S, w} S_{2}^{U S, w} S_{3}^{U S, w}\right]$

and $S_{i}^{N D I}=\left[S_{1}^{N D I} S_{2}^{N D I} S_{3}^{N D I}\right]$.

\section{Tracking spine landmarks in multiple configurations of the spine}

A set of both landmark and sensor positions in voxel coordinates relative to the center of the middle pixel of the US volume $\left(\mathrm{L}_{\text {pos }_{\mathrm{i}}}^{\mathrm{US}, \mathrm{w}}, \mathrm{S}_{\mathrm{i}}^{\mathrm{US}, \mathrm{w}}\right)$ were required for each vertebra and configuration $\operatorname{pos}_{i}$ to track the spine landmarks in NDI coordinates. As the sensors were not moved between poses during data collection, we assumed that the sensor position in voxel coordinates $\mathrm{S}_{\mathrm{i}}^{\mathrm{US}, \mathrm{w}}$ stayed constant between configurations $\operatorname{pos}_{i}$. The obtained homogeneous transformation $_{N D I} T^{U S}$ from US to NDI representation, introduced in Section II-C, was also applied to the vertebral landmarks identified in the US volumes $\left(L_{\text {pos }_{i}}^{U S, w}\right)$ to determine their location in the NDI coordinate system as follows:

$$
L_{\text {pos }_{i}}^{N D I}={ }_{N D I} T^{U S} L_{\text {pos }_{i}}^{U S, w}
$$

\section{E. Full reconstruction of the spine}

The anatomy of the thoracic spine T1-T5 was reconstructed by merging the acquired US volumes of each spine configuration ('straight', 'curved' and 'curved 2') using a semi-automatic registration procedure. Due to the limited field of view provided by the US probe, the spinal region of interest, i.e., T1-T5, could not be captured in a single US volume. Therefore, for each configuration, multiple US volumes were acquired, including a whole vertebra and parts of adjacent vertebrae. The US volumes were imported into ImFusion, enabling both the simultaneous visualization of multiple volumes in a common coordinate system and their associated homogenous transformation. Since homogeneous transformation in ImFusion maps the new coordinate system (i.e., NDI coordinate system) to the original coordinate system (i.e., US coordinate system), the inverse of the transformation matrix determined in Section II-C was applied to the respective volumes, resulting in a 'coarse' registration of the spine based on the NDI localization.

In a second step, this coarse registration was further refined using an automatic rigid registration module in ImFusion by keeping the first US volume of the vertebral sequence static while the second volume was registered to the first one. The acquired change in translation and rotation was applied to all subsequent US volumes to keep their relative position constant. Next, the third volume was registered to the second volume, and the resulting relative roto-translation applied to the subsequent volumes, and so on.

The obtained refined registrations were validated by an experienced sonographer with the possibility for modification if required and defined as the ground-truth in this study. 
$>$ REPLACE THIS LINE WITH YOUR MANUSCRIPT ID NUMBER (DOUBLE-CLICK HERE TO EDIT) <

Eventually, both reconstructions generated using the coarse and the refined registration method, respectively, were each combined in a single volume, selecting the maximum intensity voxel for the overlapping voxels of the volumes.

While in this section, the anatomical reconstruction focused on the vertebral levels T1-T5, the same registration approach can be extended to other vertebral levels of the spine. Further, we like to point out that the automatic registration was only possible given the initial coarse solution provided by the NDIbased registration.

\section{F. Error measurements}

i. The tracking method accuracy is strictly dependent upon the accuracy of sensor identification from the US volumes (Section II-B). To evaluate the accuracy of sensor identification, we computed the difference $E_{S_{i} S_{j}}$ of absolute distances between each US-derived sensor pair $S_{i} S_{j}$ with $i, \mathrm{j}=$ 1, 2, 3 and those obtained through the NDI system, as follows:

$$
\begin{gathered}
E_{S_{i} S_{j}}=D_{S_{i} S_{j}}^{N D I}-D_{S_{i} S_{j}}^{U S} \\
D_{S_{i} S_{j}}^{N D I}=\left\|S_{i}^{N D I}-S_{j}^{N D I}\right\| \\
D_{S_{i} S_{j}}^{U S}=\left\|S_{i}^{U S}-S_{j}^{U S}\right\|
\end{gathered}
$$

where $D_{S_{i} S_{j}}^{N D I}$ and $D_{S_{i} S_{j}}^{U S}$ are the absolute distances between sensor pairs obtained from the NDI system and the US volumes, respectively.

ii. The intra-observer and inter-observer variability in sensor identification was analyzed. US-derived sensor positions (sensor tips) were selected for every US volume by two observers. The intra-observer reliability in sensor selection was analyzed for a single observer (i.e., first author) and compared to the selection of another observer (i.e., second author) to obtain the inter-observer measures by computing the absolute difference between the two respective selections.

iii. The error propagation from the sensor identification to the US volumes' NDI-based coarse registration and the refined registration (i.e., how the error in the manual selection of the sensors in the US volumes propagates to the registration error) was calculated as the norm of the difference in the translation (t) and rotation (r) between the NDI-based registration (or the refined registration) and the ground-truth solution, between each two adjacent volumes [23] (10)-(11).

$$
\begin{aligned}
& t=\sqrt{\Delta \mathrm{x}^{2}+\Delta \mathrm{y}^{2}+\Delta \mathrm{z}^{2}} \\
& r=\sqrt{\Delta \alpha^{2}+\Delta \beta^{2}+\Delta \gamma^{2}}
\end{aligned}
$$

With

$$
\begin{gathered}
\Delta x=x_{R E F}-x_{N D I} ; \Delta y=y_{R E F}-y_{N D I} ; \Delta=z_{R E F}-z_{N D I} ; \\
\begin{array}{c}
\Delta \alpha=\alpha_{R E F}-\alpha_{N D I} ; \Delta \beta=\beta_{R E F}-\beta_{N D I} ; \Delta \gamma \\
=\gamma_{R E F}-\gamma_{N D I}
\end{array}
\end{gathered}
$$

where $x, y, z$ and $\alpha, \beta, \gamma$ are the three translation and rotation values, respectively, obtained by the NDI-based registration and refined registration as indicated by the subscript. The same procedure was repeated between the refined registration and the ground-truth registration.

Furthermore, the accuracy of the coarse and refined registration was quantitatively assessed by segmenting the bony anatomy through thresholding and computing the overlap between the segmentations at the intersection of adjacent US volumes. A threshold of 100 was chosen to ensure that the hyperechoic signal corresponding to the bony anatomy was included in the segmentation. The overlap between the segmentations was computed through both the Dice similarity coefficient (DSC) and the corresponding overlapping volume at the intersection of each two adjacent US volumes for the coarse and the refined registration with respect to the ground-truth solution.

\section{RESULTS}

In the present study a phantom spine model was used to develop an experimental framework to assess vertebral spinal motion by using T-US. Tracking of the US probe during data collection enabled us to establish the location of selected landmarks in a global coordinate system. To assess the accuracy of mapping landmarks validated via manual registration (Section II-B) we compared the position of each landmark in the three different configurations $\operatorname{pos}_{i}$. The absolute distance between landmarks in these configurations ranged from $0.01 \mathrm{~mm}$ and $0.56 \mathrm{~mm}$. On average, the position of landmarks varied by $0.21 \mathrm{~mm}(\mathrm{SD}=$ \pm 0.16 ). For this analysis, landmarks in the straight position were used as a reference to comply with our applied methods for landmark mapping. Further results of this study include the intra- and inter-observer reliability in sensor identification that is necessary to evaluate the suitability of US for tracking spinal landmarks using the workflow developed herein. Lastly, the performance of T-US for spinal reconstruction is assessed.

\section{A. Accuracy and reliability in sensor identification from US imaging}

Table I shows the accuracy in sensor identification as the absolute error in sensor distances between NDI-derived and US-derived sensor positions. Values ranged from $0.05 \mathrm{~mm}$ to $1.57 \mathrm{~mm}$ with an overall mean of $0.49 \mathrm{~mm}(\mathrm{SD}= \pm 0.41 \mathrm{~mm})$. The highest uncertainty was between Sensor 1 and Sensor 2 $\left(S_{1} S_{2}\right)$ with an average deviance to NDI data of $0.61 \mathrm{~mm}$.

The intra- and inter-observer reliability in sensor selection was comparable, ranging from $0.09 \mathrm{~mm}$ to $0.63 \mathrm{~mm}$ and from 0.12 $\mathrm{mm}$ to $0.57 \mathrm{~mm}$, respectively (Table II). Overall, the selection of sensor tips by the second observer showed less variance (SD range: $\pm 0.05- \pm 0.2 \mathrm{~mm}$ ) than the selection of the first observer (SD range: $\pm 0.05 \mathrm{~mm}- \pm 0.6 \mathrm{~mm}$ ). With respect to the NDIderived sensor positions, both selections showed a similar deviance from tracked sensor distances of $0.49 \mathrm{~mm}$ (Table I, first observer) and $0.58 \mathrm{~mm}$ (second observer).

\section{B. Tracking landmarks in multiple configurations of the spine}

Fig. 4 shows the three configurations pos $_{i}$ for levels T1-T5 based on US-derived spinal landmarks after being transformed into global NDI coordinates (Section II-D). Each vertebra's position and orientation is fully described by the triplet of level- 
$>$ REPLACE THIS LINE WITH YOUR MANUSCRIPT ID NUMBER (DOUBLE-CLICK HERE TO EDIT) >

specific landmarks SP, LL, LR $\in$ L forming a triangular object. These landmark triangles could successfully be used to track the two-step flexion of the phantom spine from an initially straight to a curved configuration (Fig. 4A-C).

\section{Full reconstruction of the spine in multiple configurations}

The experienced sonographer evaluated the refined registrations to be accurate (Section II-E), with no modifications needed, except for one registration which was excluded from the assessment since there was no sufficient anatomical overlap between the two adjacent US volumes in order to perform a manual or automatic registration. Therefore, we considered the refined registrations as the ground-truth in this study and used it for comparison to the NDI-based registration. As an example, Fig. 5 shows the full reconstructions of the spine in the 'straight' configuration generated by the NDI-based registration and after refinement, respectively. The average normed difference in translation and rotation between the NDI-based registration and the refined (or ground-truth) registration was $5.6 \mathrm{~mm} \pm 2.4 \mathrm{SD}$ (range: 3.3 $9.7 \mathrm{~mm}$ ) and 3.6 degrees $\pm 1.4 \mathrm{SD}$ (range: $1.3-5.2$ degrees), respectively. This result shows that the course (NDI-based) registration is on average $5.6 \mathrm{~mm}$ shifted from the ground-truth solution. The improved overlap upon refinement (or groundtruth) can be seen in Fig. 6 highlighting the intersection area of two adjacent US volumes. Table III shows the corresponding quantitative improvement of the refined registration (groundtruth) compared to the NDI-based registration (NDI reg) through the DSC and the overlapping volume computed on the bony segmentations at the intersection between each volume pair. 'Curved 2' reports the case excluded from this registration assessment where no sufficient overlap between the bony anatomy was recorded between the volumes containing T1-T3 and $\mathrm{T} 4$.

\section{DISCUSSION}

In this study we examined the potential of using 3D T-US for tracking spinal landmarks as a novel approach to assess vertebral spinal motion opposed to common marker-based techniques. US is the only imaging modality which currently allows for real-time, volumetric and dynamic assessment of the spine for healthy and pathologic conditions. Further, it is radiation-free and the most cost-effective imaging modality compatible in a standard clinical environment. The major advantage of using T-US over traditional marker-based motion capture techniques is that it enables the selection of a sufficient number of landmarks (at least three) in order to localize a body in space while at the same time overcoming limitations such as soft-tissue artifacts. In fact, marker-based techniques are known to have system errors of up to $10 \mathrm{~mm}$ when studying human movement in general [24]. Applied on the spine, even larger errors of up to $27 \mathrm{~mm}$ were observed in a forward bending [4], considering the potentially significant skin movement prevalent on the spine during motion tasks.

During the experimental setup three small electromagnetic NDI tracking sensors were attached to the US probe and later identified in the US volumes in order to obtain information on the global position and orientation of vertebrae. The localization of the probe including sensor identification was the part of the developed workflow most prone to error. As we cannot assess each sensor's position individually and the error resulting from it, the relative distance between US-derived sensor positions was computed and compared to NDI sensor data. Our results in Table I and Table II show a high reliability in sensor identification as the error measured between USderived sensor tips and tracked sensor positions was small ( 0.49 $\mathrm{mm}$ on average). The largest intra-observer variability in sensor selection was $1.2 \mathrm{~mm}$ (Table II) while the inter-observer variability was strongly correlated to the intra-observer data with a maximum deviance of $0.8 \mathrm{~mm}$, proving that our methodology is transferable between observers. An important factor contributing to the inter-observer variability is that, as a consequence of the high resolution of US images, the sensor tips were visible in multiple adjacent voxels (voxel dimension: $0.18 \mathrm{~mm} \times 0.18 \mathrm{~mm} \times 0.34 \mathrm{~mm}$ ).

Regarding landmark identification, automatic solutions could be considered in future [18,25], however, these techniques have shown limited success in terms of a robust extraction of the lamina given that it covers a broad area of the vertebra. Hence, for tracking purposes where exact landmark positions are crucial, i.e., for inverse kinematic simulations, mapping of landmarks, like the one proposed in this paper, should be preferred over a landmark reselection. This is supported by the small relative distance error of $0.21 \mathrm{~mm}(\mathrm{SD}= \pm 0.16)$ produced by our method on average that mapped landmarks initially selected in the 'straight' configuration to the other configurations ('curved', 'curved 2'). Although considered to be of negligible magnitude, a possible explanation for this error may be that the desired landmark was placed at the border of two adjacent voxels and was then shifted to the voxels' center coordinates. As no MRI-derived information was further used in our workflow, the time-consuming endeavor of US-MRI registration for landmark validation could potentially be avoided in future if the observer is familiar with spinal anatomy in US and confident in selecting landmarks. Alternatively, automatic registration algorithms could be taken into consideration [26]. In our opinion, 3D US is crucial for a correct identification of landmarks considering the manifestation of the spinous process particularly in the thoracic region that results in a partial overlap of vertebral levels. In the transverse plane, hence, prominent bony features may belong to different vertebrae and could lead to a misinterpretation of a $2 \mathrm{D}$ US image.

Based on the validation by an experienced sonographer, our approach of NDI-derived registration followed by the refined automatic registration together created full US-based spinal reconstructions comparable to clinical standards, hence, accepting our second hypothesis that 3D T-US is suitable for an accurate reconstruction of the (phantom) spine. A challenge related to the refined registration algorithm used in this study is that although robust to artifacts generated by water motion, and the plastic tank enclosing the spine surrogate, this method is likely reserved for phantom studies that provide a strong contrast and simplified anatomy. In-vivo, surrounding softtissue will influence the signal reflection intensity of US and cause additional artifacts, increasing the complexity of the problem. Other registration methods, most likely deep learning algorithms specifically trained for this application, will need to be introduced. Moreover, an initial coarse registration of US 
$>$ REPLACE THIS LINE WITH YOUR MANUSCRIPT ID NUMBER (DOUBLE-CLICK HERE TO EDIT) <

volumes was required for the used automatic registration algorithm. As mentioned in Section III-C, there was not enough overlap between the US volumes covering T1-T3/T4 in the 'curved 2' configuration to perform the automatic registration, hence, it was excluded from evaluation. To overcome this problem in future, smaller increments along the spine would be advisable during scanning to obtain good coverage and sufficient overlap between the scans. Evaluation of the remaining coarse registrations led to a propagation error of 5.6 $\mathrm{mm}$ from the refined registration which is significantly lower than the aforementioned system errors reported in marker-based techniques on the spine [4]. Two factors were identified to contribute to this propagation error, that is, optimization-related inaccuracies introduced to determine the US to NDI transformation matrix ${ }_{N D I} T^{U S}$ (Section II-C) and the variability in sensor identification. The latter could be eliminated while significantly accelerating the proposed workflow if the US system featured an integrated localization of the probe as in [27].

To assess the validity of our approach in tracking spinal landmarks the same propagation error applies as for the comparison of the coarse registration to the refined (groundtruth) registration. This is because the transformation of landmarks from US to NDI coordinates (6) underlies the same transformation matrix as determined in Section II-C to create the coarse registration. Considering the significant improvement with respect to marker-based approaches, we accept our first hypothesis that 3D T-US allows tracking of spinal landmarks.

Ultimately, with respect to intervertebral motion, knowing the global position and orientation of each vertebra based on the information obtained from its landmarks the relative change in vertebral translation and rotation could be evaluated by reapplying the least-square optimization from Section II-C on two sets (triplets) of landmarks in global NDI coordinates. Given a phantom spine was used in the current study, we found this to be irrelevant, however, in future work considering physiological spinal movement, information on flexion kinematics would be highly valuable for the biomechanics research community.

A limitation of this proof-of-concept study is that only static postures were examined. In future, the proposed methodology should be extended to dynamic analysis of spinal motion and applied on human participants. Ideally, a participant could perform movements while a tracked US probe is attached to the participant's back scanning the vertebra's posterior surface in real-time. The process could then be repeated multiple times to allow for full coverage of the whole spine. Or, in more advanced scenarios, multiple probes or even distributed flexible large scanning arrays could be considered at the same time, for example using the novel capacitive micromachined ultrasound transducers (CMUTs) [28] or the piezoelectric micromachined ultrasound transducers (PMUTs) [29].

\section{CONCLUSION}

Summarizing, the present study has shown that 3D T-US is a precise tool for the selection and tracking of vertebral landmarks and holds the potential for advancing motion capture of the spine. Thereby T-US promotes the functional capability to provide insight into internal spine kinematics which could overcome limitations of current gold standards.

\section{REFERENCES}

[1] A. C. Breen et al., "Measurement of intervertebral motion using quantitative fluoroscopy: report of an international forum and proposal for use in the assessment of degenerative disc disease in the lumbar spine," Advances in orthopedics, vol. 2012, 2012.

[2] J. Dvořák, M. M. Panjabi, D. G. Chang, R. Theiler, and D. Grob, "Functional radiographic diagnosis of the lumbar spine: flexion-extension and lateral bending," Spine, vol. 16, no. 5, pp. 562-571, 1991.

[3] M. J. Pearcy and M. W. Whittle, "Movements of the lumbar spine measured by three-dimensional X-ray analysis," Journal of biomedical engineering, vol. 4, no. 2, pp. 107-112, 1982.

[4] R. Zemp et al., "Soft tissue artefacts of the human back: comparison of the sagittal curvature of the spine measured using skin markers and an open upright MRI," PloS one, vol. 9, no. 4, p. e95426, 2014.

[5] S. Mahallati, H. Rouhani, R. Preuss, K. Masani, and M. R. Popovic, "Multisegment kinematics of the spinal column: soft tissue artifacts assessment," Journal of biomechanical engineering, vol. 138, no. 7, p. 071003, 2016.

[6] A. A. White and M. M. Panjabi, "The basic kinematics of the human spine. A review of past and current knowledge.," Spine, vol. 3, no. 1, pp. 12-20, 1978.

[7] A. G. Bruno, M. L. Bouxsein, and D. E. Anderson, "Development and validation of a musculoskeletal model of the fully articulated thoracolumbar spine and rib cage," Journal of biomechanical engineering, vol. 137, no. 8, p. 081003, 2015.

[8] A. Seth et al., "OpenSim: Simulating musculoskeletal dynamics and neuromuscular control to study human and animal movement," PLoS computational biology, vol. 14, no. 7, p. e1006223, 2018.

[9] J. R. Daniell and O. L. Osti, "Failed back surgery syndrome: a review article," Asian spine journal, vol. 12, no. 2, pp. 372-379, 2018.

[10] D. MacDonald, G. L. Moseley, and P. W. Hodges, "People with recurrent low back pain respond differently to trunk loading despite remission from symptoms," Spine, vol. 35, no. 7, pp. 818-824, 2010.

[11] W. Chen, E. H. M. Lou, and L. H. Le, "Using ultrasound imaging to identify landmarks in vertebra models to assess spinal deformity," in 2011 Annual International Conference of the IEEE Engineering in Medicine and Biology Society, 2011, pp. 8495-8498.

[12] S. Suzuki, T. Yamamuro, J. Shikata, K. Shimizu, and H. Iida, "Ultrasound measurement of vertebral rotation in idiopathic scoliosis," The Journal of bone and joint surgery. British volume, vol. 71, no. 2, pp. 252-255, 1989. 
> REPLACE THIS LINE WITH YOUR MANUSCRIPT ID NUMBER (DOUBLE-CLICK HERE TO EDIT) <

[13] Y. S. Wong et al., "Is radiation-free ultrasound accurate for quantitative assessment of spinal deformity in idiopathic scoliosis (IS): a detailed analysis with EOS radiography on 952 patients," Ultrasound in medicine \& biology, vol. 45, no. 11, pp. 2866-2877, 2019.

[14] S. de Reuver et al., "Cross-validation of ultrasound imaging in adolescent idiopathic scoliosis," European Spine Journal, vol. 30, no. 3, pp. 628-633, 2021.

[15] T. Ungi et al., "Spinal curvature measurement by tracked ultrasound snapshots," Ultrasound in medicine \& biology, vol. 40, no. 2, pp. 447-454, 2014.

[16] H.-B. Chen et al., "Improvement of 3D Ultrasound Spine Imaging technique using Fast Reconstruction Algorithm," IEEE Transactions on Ultrasonics, Ferroelectrics, and Frequency Control, vol. 68, no. 10, pp. 3104-3113, 2021.

[17] W. van den Hoorn, M. W. Coppieters, J. H. van Dieën, and P. W. Hodges, "Development and validation of a method to measure lumbosacral motion using ultrasound imaging," Ultrasound in medicine \& biology, vol. 42, no. 5, pp. 1221-1229, 2016.

[18] A. Brignol, H.-E. Gueziri, F. Cheriet, D. L. Collins, and C. Laporte, "Automatic extraction of vertebral landmarks from ultrasound images: A pilot study," Computers in Biology and Medicine, vol. 122, p. 103838, 2020.

[19] K. Niu, V. Sluiter, J. Homminga, A. Sprengers, and N. Verdonschot, "A novel ultrasound-based lower extremity motion tracking system," in Intelligent Orthopaedics.: Springer, 2018, vol. 1093, pp. 131-142.

[20] K. Niu et al., "In situ comparison of A-mode ultrasound tracking system and skin-mounted markers for measuring kinematics of the lower extremity," Journal of biomechanics, vol. 72, pp. 134-143, 2018.

[21] M. A. Masum, M. Pickering, A. Lambert, J. Scarvell, and P. Smith, "Accuracy assessment of Tri-plane Bmode ultrasound for non-invasive 3D kinematic analysis of knee joints," Biomedical engineering online, vol. 13, no. $122,2014$.

[22] J. Matt. (2021) Absolute Orientation - Horn's method. [Online].

https://www.mathworks.com/matlabcentral/fileexchange/ 26186-absolute-orientation-horn-s-method

[23] M. Antico et al., "4D Ultrasound-based knee joint atlas for robotic knee arthroscopy: a feasibility study," IEEE Access, vol. 8, pp. 146331-146341, 2020.

[24] J. L. Hicks, T. K. Uchida, A. Seth, A. Rajagopal, and S. L. Delp, "Is my model good enough? Best practices for verification and validation of musculoskeletal models and simulations of movement," Journal of biomechanical engineering, vol. 137, no. 2, p. 020905 , 2015.

[25] H.-Y. Zeng, E. Lou, S.-H. Ge, Z.-C. Liu, and R. Zheng, "Automatic Detection and Measurement of Spinous Process Curve on Clinical Ultrasound Spine Images," IEEE Transactions on Ultrasonics, Ferroelectrics, and Frequency Control, vol. 68, no. 5, pp. 1696-1706, 2020.
[26] T. K. Koo, R. L. Crews, and W. E. Kwok, "In Vivo Measurement of the Human Lumbar Spine Using Magnetic Resonance Imaging to Ultrasound Registration," Journal of manipulative and physiological therapeutics, vol. 42, no. 5, pp. 343-352, 2019.

[27] M. Lachaine and T. Falco, "Intrafractional prostate motion management with the Clarity Autoscan system," Med Phys Int J, vol. 1, no. 1, pp. 72-80, 2013.

[28] O. Oralkan et al., "Capacitive micromachined ultrasonic transducers: Next-generation arrays for acoustic imaging?," IEEE transactions on ultrasonics, ferroelectrics, and frequency control, vol. 49, no. 11, pp. 1596-1610, 2002.

[29] P. Muralt et al., "Piezoelectric micromachined ultrasonic transducers based on PZT thin films," IEEE transactions on ultrasonics, ferroelectrics, and frequency control, vol. 52, no. 12, pp. 2276-2288, 2005. 


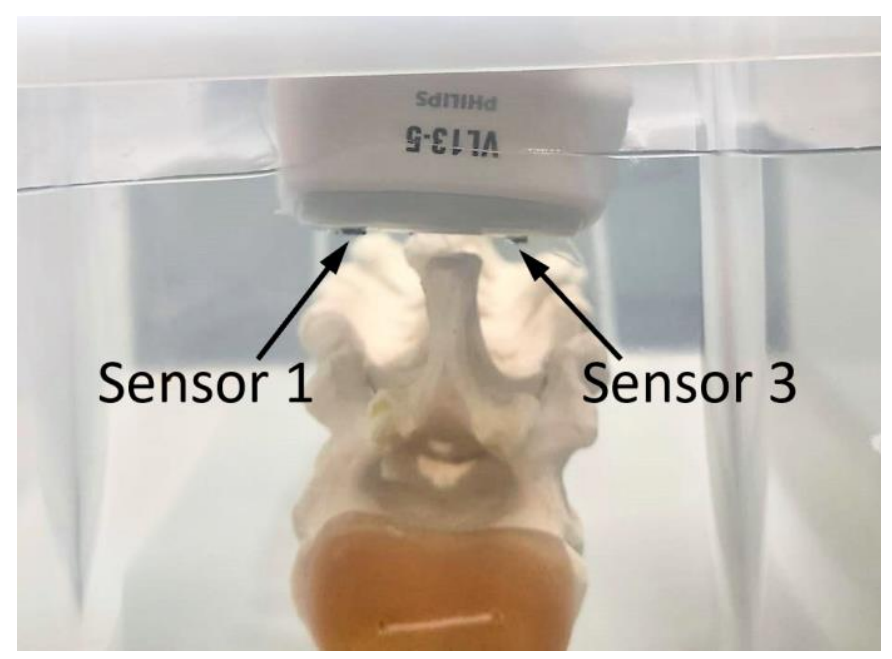

Fig. 1. Picture of the experimental data collection. The phantom spine immersed in water was scanned using US in different configurations while three electromagnetic sensors were attached to the probe head and tracked through the NDI system. Visible sensors (here: Sensor 1 and Sensor 3) are indicated

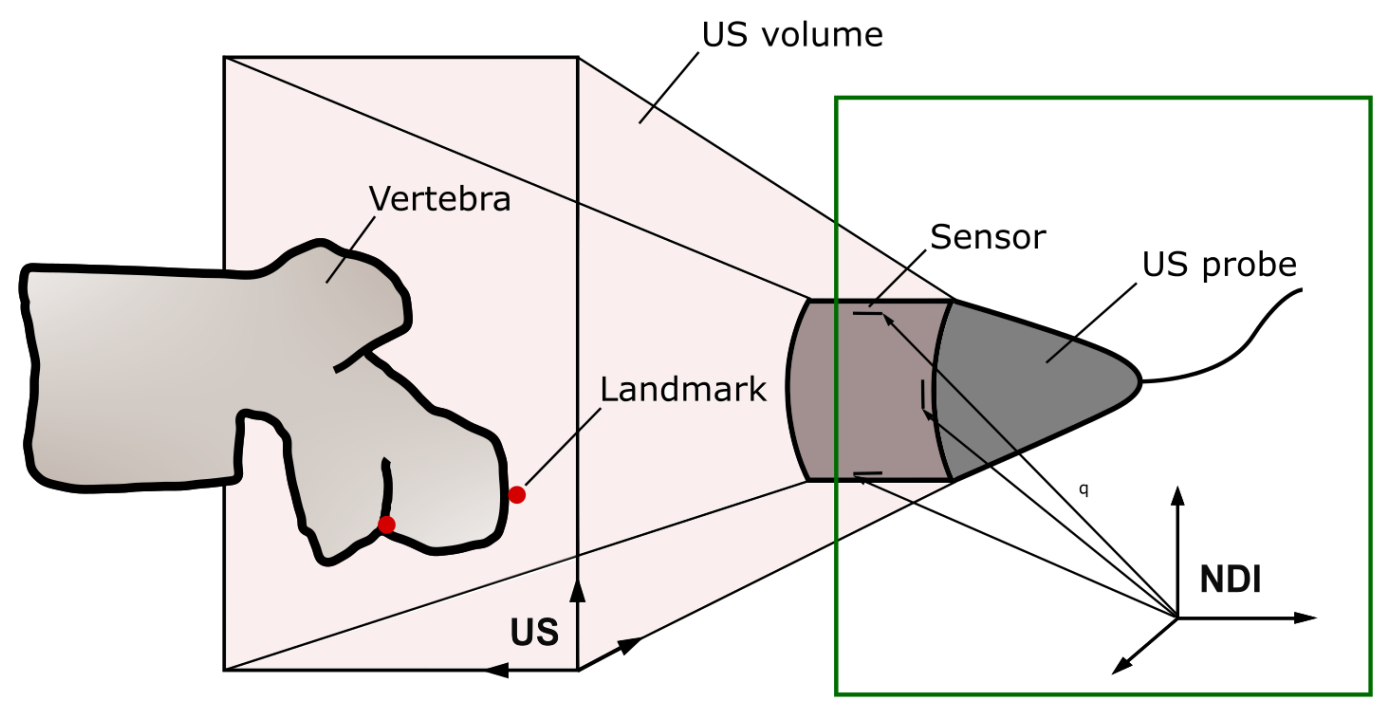

Fig. 2. Illustration of the experimental data collection using T-US. The location of the US probe was tracked by an electromagnetic tracking system (NDI). Every voxel within the US volume (red area) created by the 3D US transducer can be related to the location of the tracking sensors attached to the probe head. Landmarks selected on the scanned vertebra can be identified and located in the NDI reference system using coordinate transformation (Section II-D) 

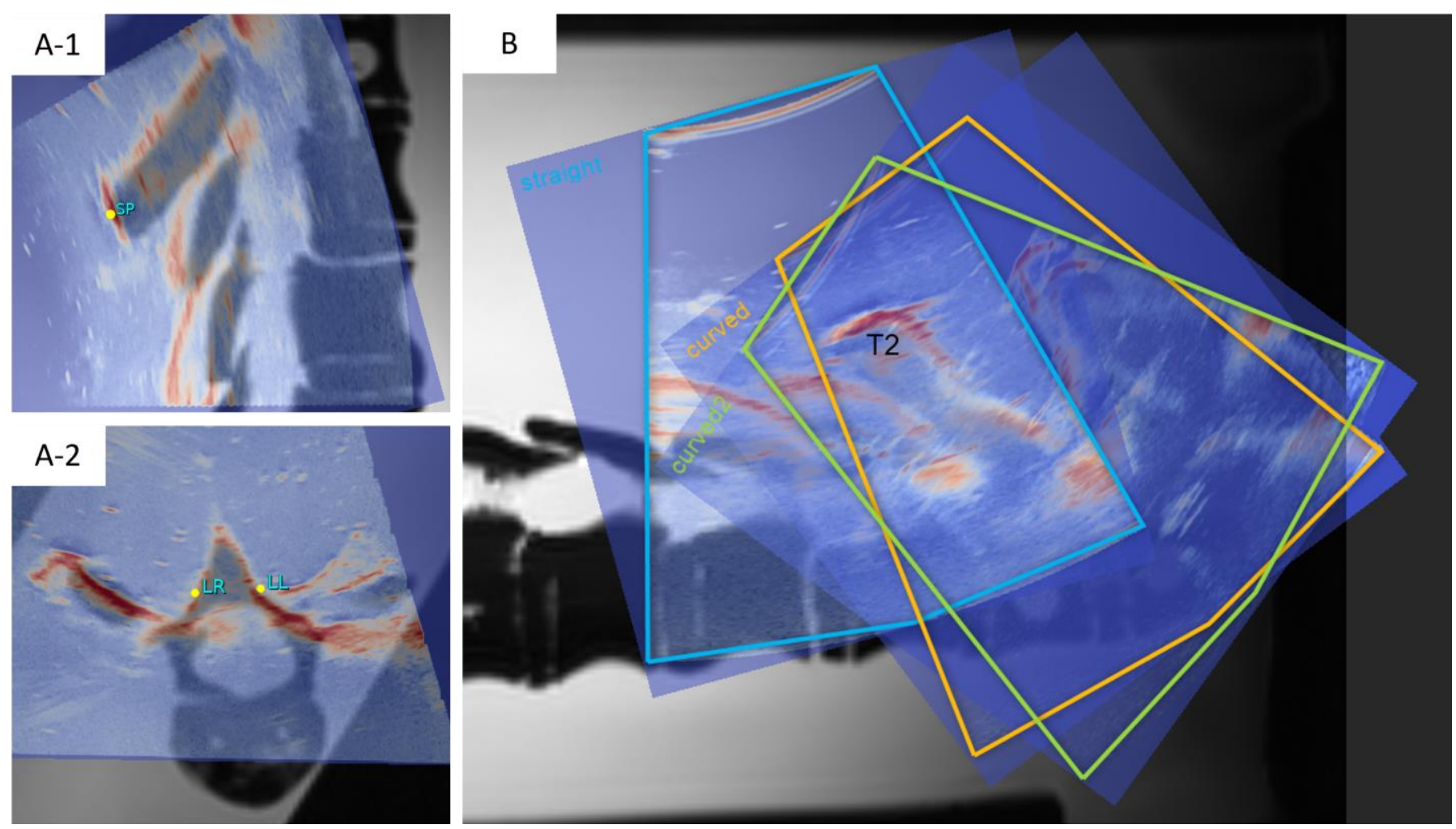

Fig. 3. Manual US-MRI registration for vertebra T2 in the sagittal (A-1) and axial plane (A-2). Areas of strong US reflection are the SP and the laminae (indicated) on both anatomical sides (left: LL, right: LR). The selection of landmarks was verified by manual US-MRI image registration. In B, US-MRI registration is visualized for T2 in all spine configurations pos $_{i}$ with $\mathrm{i}=1,2$, 3. US-derived sensor tips $S_{\text {pos }_{i}}^{U S}$ were selected individually for every US volume (straight: blue, curved: orange, curved 2: green) while the triplet of landmarks $L_{\text {pos }_{i}}^{U S}$ was mapped from the straight configuration to the curved configurations according to (1) 
$>$ REPLACE THIS LINE WITH YOUR MANUSCRIPT ID NUMBER (DOUBLE-CLICK HERE TO EDIT) <
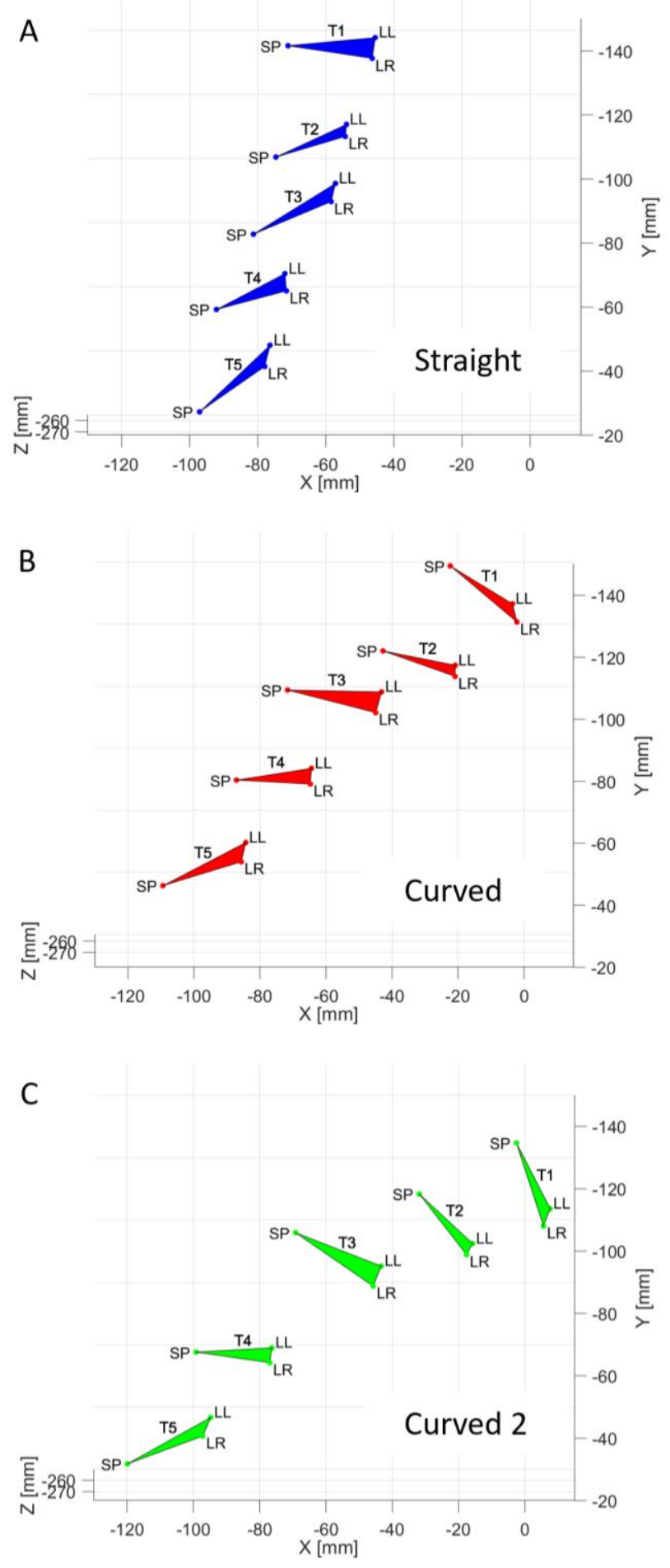

Fig. 4. Tracking US-derived spinal landmarks in three configurations (A: Straight, B: Curved, C: Curved 2). The level-specific triplet of landmarks fully describes a vertebra's position in global NDI coordinates. Landmarks were selected for T1-T5 in the 'straight' configuration and mapped to the curved configurations after US-MRI registration verified their precise selection 

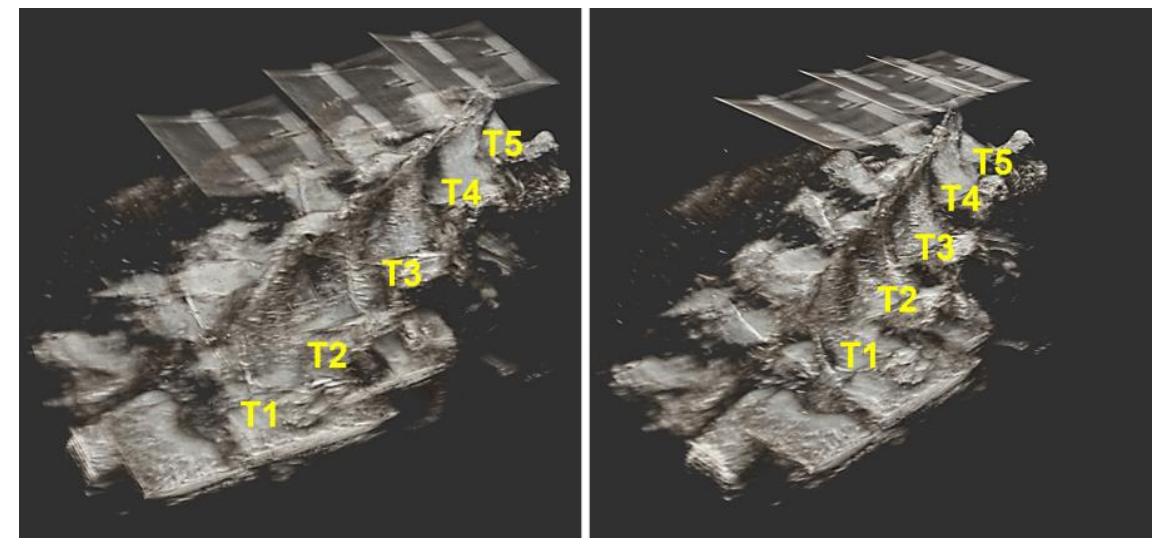

Fig. 5. Example of a full reconstruction. 3D rendering of the thoracic spine (T1-T5) in the 'straight' configuration generated through the NDI-based registration (left) and the refined registration (right). The vertebral levels T1-T5 are indicated in yellow
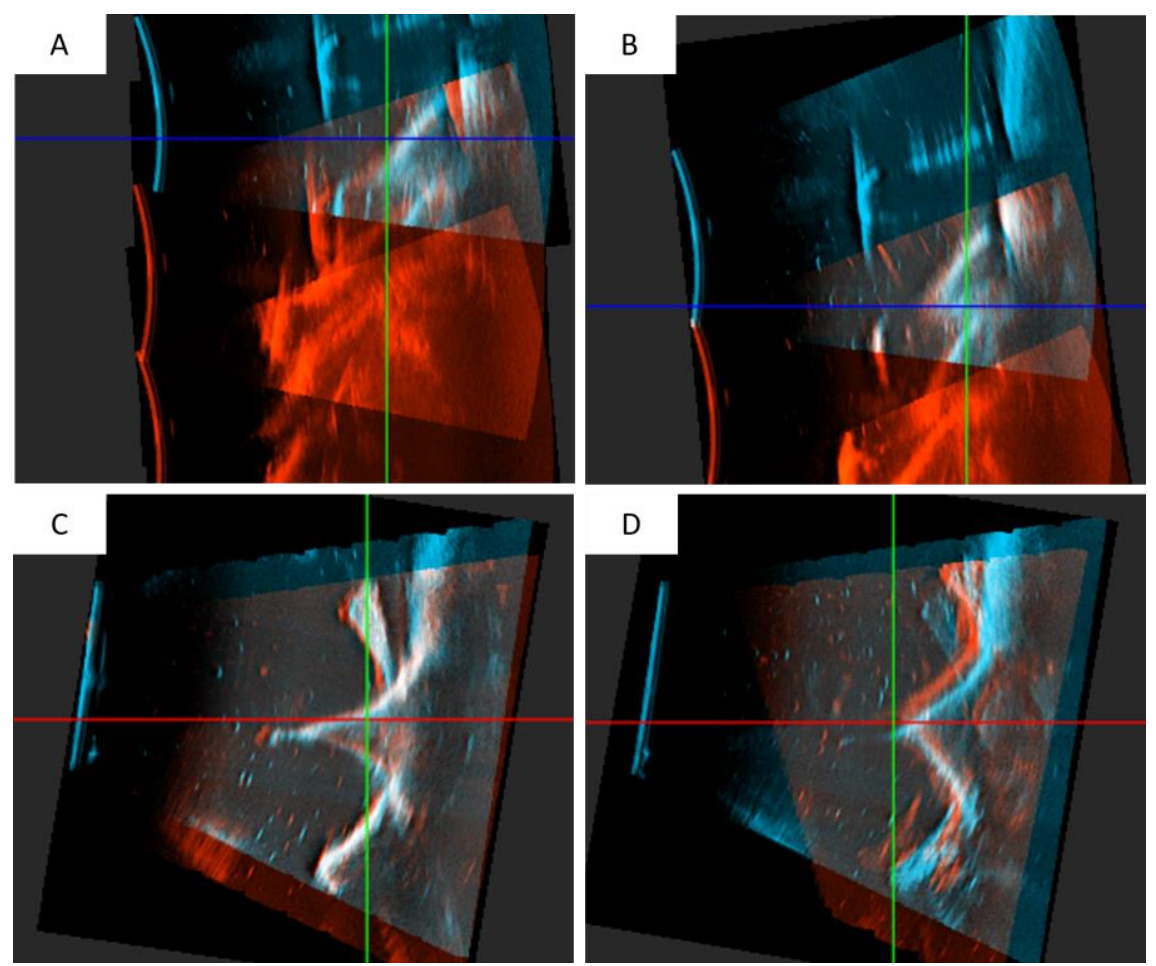

Fig. 6. Example of the overlap between the bony anatomy in the intersecting area of two US volumes merged through the NDIbased coarse registration (A and C) and the refined registration (B and D), shown along the sagittal (A and B) and the axial plane (C and D). The two adjacent US volumes are visualized in blue and red while the overlapping regions are shown in white 
$>$ REPLACE THIS LINE WITH YOUR MANUSCRIPT ID NUMBER (DOUBLE-CLICK HERE TO EDIT) <

TABLE I

ABSOLUTE ERROR IN SENSOR DISTANCES BETWEEN

NDI-DERIVED AND US-DERIVED SENSOR POSITIONS

\begin{tabular}{llll}
\hline \multicolumn{1}{r}{ pos $_{i}$} & \multicolumn{3}{c}{ Absolute distance error } \\
& $S_{1} S_{3}[\mathrm{~mm}]$ & $S_{1} S_{2}[\mathrm{~mm}]$ & $S_{2} S_{3}[\mathrm{~mm}]$ \\
\hline straight & & & \\
$\mathrm{T} 1$ & 0.7 & 0.72 & 0.96 \\
$\mathrm{~T} 2 / 3$ & 0.3 & 0.24 & 0.18 \\
$\mathrm{~T} 4 / 5$ & 0.08 & 0.96 & 0.14 \\
mean $( \pm \mathrm{SD})$ & $0.36(0.31)$ & $0.64(0.37)$ & $0.43(0.46)$ \\
curved & & & \\
T1/2 & 0.13 & 0.08 & 0.4 \\
T3/4 & 0.19 & 0.25 & 0.85 \\
T5 & 0.2 & 0.88 & 1.33 \\
mean $( \pm \mathrm{SD})$ & $0.17(0.04)$ & $0.4(0.42)$ & $0.86(0.47)$ \\
curved 2 & & & \\
T1-T3 & 0.44 & 0.29 & 0.11 \\
T4 & 0.89 & 1.57 & 0.05 \\
T5 & 0.3 & 0.56 & 0.49 \\
mean $( \pm \mathrm{SD})$ & $0.54(0.31)$ & $0.8(0.67)$ & $0.22(0.24)$ \\
\hline overall mean & $0.49(0.41)$ & & \\
$( \pm \mathrm{SD})$ & & & \\
\hline
\end{tabular}

TABLE II

INTRA- AND INTER-OBSERVER RELIABILITY IN SENSOR SELECTION USING US

\begin{tabular}{|c|c|c|c|c|c|c|c|c|c|}
\hline \multirow{2}{*}{\multicolumn{2}{|c|}{$\frac{\operatorname{pos}_{i} \quad \text { Sensor } 1}{\text { Intra-observer reliabil }}$}} & & & \multicolumn{3}{|c|}{ Sensor 2} & \multicolumn{3}{|c|}{ Sensor 3} \\
\hline & & (single & erver)* & & & & & & \\
\hline \multirow{3}{*}{$\begin{array}{l}\text { mean } \\
( \pm \mathrm{SD})\end{array}$} & $x$ & $y$ & $z$ & $x$ & $y$ & $z$ & $x$ & $y$ & $z$ \\
\hline & 0.27 & 0.15 & 0.57 & 0.24 & 0.18 & 0.17 & 0.09 & 0.21 & 0.63 \\
\hline & $(0.16)$ & $(0.14)$ & $(0.43)$ & $(0.05)$ & $(0.24)$ & $(0.3)$ & $(0.09)$ & $(0.14)$ & $(0.6)$ \\
\hline straight & 0.09 & 0.27 & 0.51 & 0.27 & 0.46 & 0 & 0.09 & 0.36 & 0.68 \\
\hline curved & 0.36 & 0 & 0.17 & 0.18 & 0 & 0 & 0.18 & 0.18 & 1.2 \\
\hline curved 2 & 0.36 & 0.18 & 1.03 & 0.27 & 0.09 & 0.51 & 0 & 0.09 & 0 \\
\hline \multicolumn{10}{|c|}{ Inter-observer reliability (two observers)* [mm] } \\
\hline & $x$ & $y$ & $z$ & $x$ & $y$ & $z$ & $x$ & $y$ & $z$ \\
\hline $\begin{array}{l}\text { mean } \\
( \pm \mathrm{SD})\end{array}$ & $0.14(0.09)$ & $\begin{array}{l}0.57 \\
(0.07)\end{array}$ & $\begin{array}{l}0.49 \\
(0.13)\end{array}$ & $\begin{array}{l}0.12 \\
(0.06)\end{array}$ & $\begin{array}{l}0.18 \\
(0.06)\end{array}$ & $\begin{array}{l}0.23 \\
(0.2)\end{array}$ & $\begin{array}{l}0.16 \\
(0.04)\end{array}$ & $\begin{array}{l}0.2 \\
(0.09)\end{array}$ & $\begin{array}{l}0.57 \\
(0.3)\end{array}$ \\
\hline straight & 0.12 & 0.61 & 0.34 & 0.06 & 0.18 & 0.34 & 0.18 & 0.3 & 0.23 \\
\hline curved & 0.06 & 0.61 & 0.57 & 0.12 & 0.12 & 0 & 0.12 & 0.18 & 0.8 \\
\hline curved 2 & 0.24 & 0.49 & 0.57 & 0.18 & 0.24 & 0.34 & 0.18 & 0.12 & 0.68 \\
\hline
\end{tabular}

*All values are given in $\mathrm{mm}$ 
$>$ REPLACE THIS LINE WITH YOUR MANUSCRIPT ID NUMBER (DOUBLE-CLICK HERE TO EDIT) <

TABLE III

IMPROVEMENT OF THE REFINED REGISTRATION COMPARED TO THE NDI-DERIVED REGISTRATION

\begin{tabular}{|c|c|c|c|c|c|}
\hline \multirow[t]{2}{*}{$\operatorname{pos}_{i}$} & \multicolumn{2}{|c|}{ Abs DSC [\%] * } & \multirow{2}{*}{$\begin{array}{c}\text { Rel DSC [\%] * } \\
\text { Abs DSC (NDI reg) / Abs DSC } \\
\text { (Refined reg or ground-truth) }\end{array}$} & \multicolumn{2}{|c|}{ Volume overlap $\left[\mathrm{cm}^{3}\right]$} \\
\hline & NDI reg & $\begin{array}{l}\text { Refined reg or } \\
\text { ground-truth }\end{array}$ & & NDI reg & $\begin{array}{l}\text { Refined reg or } \\
\text { ground-truth }\end{array}$ \\
\hline \multicolumn{6}{|l|}{ Straight } \\
\hline $\mathrm{T} 1-\mathrm{T} 2 / 3$ & 15.6 & 26.1 & 60 & 8.2 & 14.1 \\
\hline $\mathrm{T} 2 / 3-\mathrm{T} 4 / 5$ & 15.7 & 21 & 75 & 6.3 & 9.3 \\
\hline \multicolumn{6}{|l|}{ Curved } \\
\hline $\mathrm{T} 1 / 2-\mathrm{T} 3 / 4$ & 4.5 & 11.5 & 39 & 1.9 & 4.7 \\
\hline T3/4 - T5 & 1.4 & 3.5 & 40 & 0.5 & 1.3 \\
\hline \multicolumn{6}{|l|}{ Curved 2} \\
\hline $\mathrm{T} 1-\mathrm{T} 3 / \mathrm{T} 4$ & - & - & - & - & - \\
\hline $\mathrm{T} 4$ - T5 & $6.1(37)$ & 16.7 & 37 & 0.8 & 2.2 \\
\hline
\end{tabular}

*Absolute (Abs) and relative (Rel) DSC, computed as the DSC obtained by the NDI reg with respect to the maximum DSC achievable, defined as the DSC obtained for the refined or ground-truth registrations.

The volume containing T1-T3/T4 in the 'curved 2' configuration was excluded from this measurement due to insufficient overlap 